\title{
6.1. SUR L'UTILISATION DES DIFFERENCES DE LONGITUDE POUR LA DÉTERMINATION \\ DU PÔLE INSTANTANÉ
}

A. Gougenheim

(Paris, France)

\section{RÉSUMÉ}

Pour déterminer la position du pôle instantané, on peut recourir, en dehors des méthodes de calcul, à une méthode graphique que l'on décrit.

\section{ABSTRACT}

To determine the position of the instantaneous pole one may use, in addition to calculations, a graphical method, which is described.

1. La méthode des moindres carrés, utilisée d'une manière générale dans le cas d'observations surabondantes exige que l'on rende d'abord linéaires les équations d'observation à l'aide d'une solution approchée. S'il n'y a que deux inconnues, ce qui est le cas des déterminations de position, les équations rendues linéaires peuvent être représentées sur un graphique à grande échelle par des droites qui sont les tangentes aux lieux géométriques correspondant aux diverses observations et dont le point de concours fournit la solution du problème. En général les droites ne concourent pas exactement et présentent une certaine dispersion due, partie aux erreurs d'observation, partie à l'incertitude affectant les éléments de base. Si l'on est gêné par cette dispersion pour adopter la solution, on peut toujours recourir à la méthode des moindres carrés, mais le graphique reste utile par la vue synthétique qu'il donne des observations et par l'approximation qu'il laisse prévoir pour le résultat.

2. Dès 1960, M. André Danjon avait montré qu'un tel graphique pouvait être établi pour la détermination du pôle instantané à l'aide de mesures de latitude. Une droite de latitude est perpendiculaire au méridien de la station d'observation et passe à une distance du pôle de référence, pris comme point approché, égale à la différence $\Delta \varphi$ entre la latitude mesurée et la latitude conventionnelle de la station.

3. Mais on peut également utiliser de la même façon les mesures de différence de longitude. Le lieu géométrique correspondant à une mesure est un segment capable sphérique décrit sur la base formée par les zéniths des deux stations en cause. Les

Markowitz and Guinot (eds.), Continental Drift, 95-97. () I.A.U. 


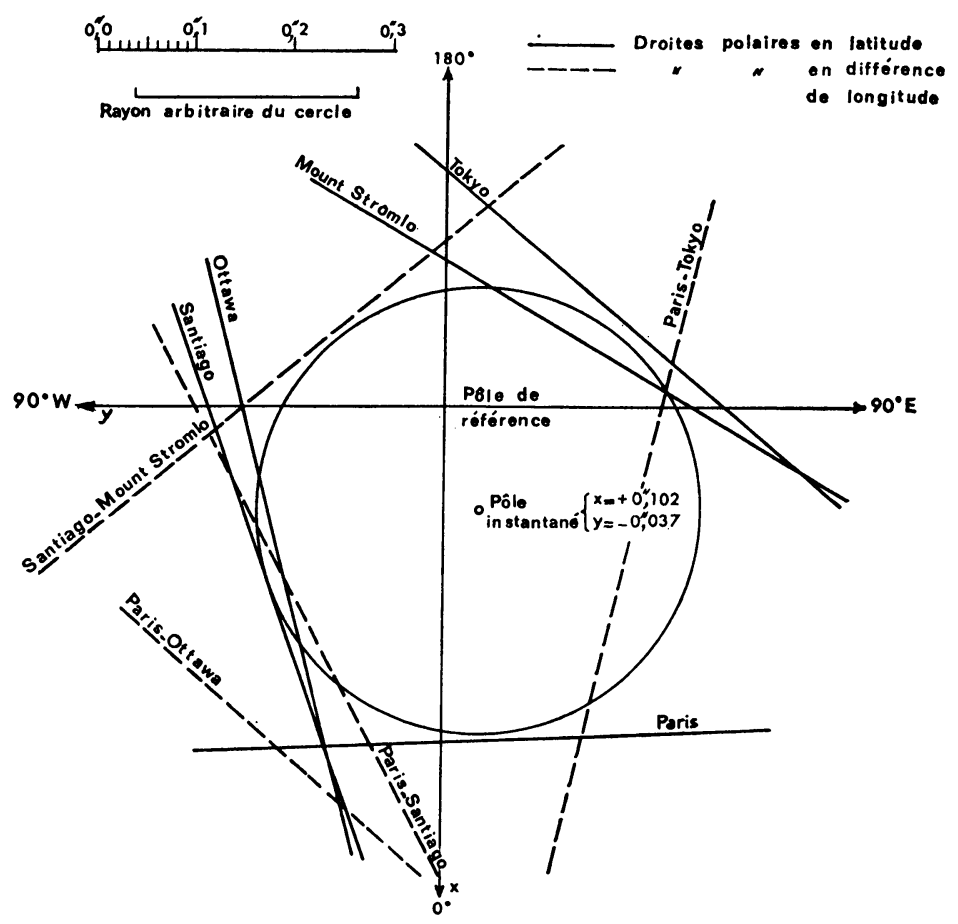

FIG. 1.

propriétés du segment capable sphérique montrent que le méridien du vertex de la base fait avec le méridien d'une des stations un angle égal à celui que le méridien de l'autre station fait avec le méridien normal au lieu, d'où la direction de celui-ci. En outre, si l'on appelle $\Delta G$ le petit écart entre la différence de longitude mesurée et la valeur conventionnelle qui correspond au pôle de référence, le lieu géométrique passe à une distance de ce dernier point égale au produit de $\Delta G$ par la cosécante de la différence de longitude et par la cotangente de la latitude du vertex.

La dispersion des divers lieux obtenus provient des erreurs d'observation, des erreurs des catalogues d'étoiles, et aussi, mais pour une très faible part, de la variation éventuelle des longitudes conventionnelles sous diverses actions, parmi lesquelles peut-être une dérive des continents.

4. Si les systèmes de latitudes conventionnelles et de longitudes conventionnelles correspondent exactement à un même pôle de référence, on peut faire concourir les droites de latitude et les droites de différence de longitude à la détermination du pôle instantané.

C'est dans cet esprit qu'a été établi l'exemple figurant sur la planche jointe, à l'aide de valeurs des coordonnées conventionnelles et des éléments d'observation de cinq 
stations; ces valeurs qui m'ont aimablement été communiquées par M. Bernard Guinot, directeur du Bureau International de l'Heure, se rapportent à une époque récente. Le graphique comporte cinq droites de latitude et quatre de différence de longitude. Sept de ces neuf lieux géométriques passent à l'intérieur d'une circonférence de 0 ".03 de rayon. Aussi pour faciliter la résolution tous les lieux géométriques ont été déplacés parallèlement à eux-mêmes d'une quantité arbitraire $(0$ ".225) de sorte que la résolution graphique consiste à déterminer le centre d'une circonférence de 0 ".225 de rayon et tangentant au mieux l'ensemble des lieux géométriques. 